\title{
Improving Clinical Performance of an Interprofessional Emergency Medical Team through a One-day Crisis Resource Management Training
}

\author{
Teodora Sorana Truta ${ }^{1}$, Cristian Marius Boeriu1 ${ }^{*}$, Marc Lazarovici², Irina Ban ${ }^{3}$, \\ Marius Petrişor ${ }^{1}$, Sanda-Maria Copotoiu ${ }^{1}$ \\ 1 University of Medicine and Pharmacy Tirgu-Mures, Street Gheorghe Marinescu 38, 540139 Tirgu-Mures, Mures, \\ Romania \\ 2 Institut für Notfallmedizin und Medizinmanagement- INM, Klinikum der Universität, Ludwig-Maximilains-Universität \\ München, Schillestraße 53, 80336 München, Germany \\ 3 Department of Medicine University of Padova, Via V. Galluci, 13-35121, Padova, Italy
}

\begin{abstract}
Introduction: Errors are frequent in health care and Emergency Departments are one of the riskiest areas due to frequent changes of team composition, complexity and variety of the cases and difficulties encountered in managing multiple patients. As the majority of clinical errors are the results of human factors and not technical in nature or due to the lack of knowledge, a training focused on these factors appears to be necessary. Crisis resource management (CRM), a tool that was developed initially by the aviation industry and then adopted by different medical specialties as anesthesia and emergency medicine, has been associated with decreased error rates.

The aim of the study: To assess whether a single day CRM training, combining didactic and simulation sessions, improves the clinical performance of an interprofessional emergency medical team.

Material and Methods: Seventy health professionals with different qualifications, working in an emergency department, were enrolled in the study. Twenty individual interprofessional teams were created. Each team was assessed before and after the training, through two in situ simulated exercises. The exercises were videotaped and were evaluated by two assessors who were blinded as to whether it was the initial or the final exercise. Objective measurement of clinical team performance was performed using a checklist that was designed for each scenario and included essential assessment items for the diagnosis and treatment of a critical patient, with the focus on key actions and decisions. The intervention consisted of a one-day training, combining didactic and simulation sessions, followed by instructor facilitated debriefing. All participants went through this training after the initial assessment exercises.

Results: An improvement was seen in most of the measured clinical parameters.

Conclusion: Our study supports the use of combined CRM training for improving the clinical performance of an interprofessional emergency team. Empirically this may improve the patient outcome.
\end{abstract}

Keywords: interprofessional, clinical performance, training, crisis resource management, emergency department

Received: 18 August 2018 / Accepted: 08 September 2018

\section{INTRODUCTION}

In the recent years, the need to enhance patient safety has become a priority as the majority of clinical errors (approximately $70 \%$ ) are the results of human factors [1] and not technical in nature or due to the lack of knowledge (failure in team communication, situational awareness, resource utilization and leadership) [2]. Ef- forts are necessary to improve the quality of the medical care provided and the patient outcome, especially in high risk areas as emergency medicine, anesthesia, surgery and intensive care.

How can we achieve this if just improving the knowledge is not enough? Steps were taken by adopting and adapting the Crises Resource Management (CRM) training, a tool that is oriented towards human 
factors and was initially developed by the aviation industry. CRM training is considered to be responsible for the decrease in aircraft accidents over the last four decades [3].

The primary objectives of CRM training are to improve team dynamics, to identify and help change mental models that create barriers in adopting effective communication, effective task management, healthy leadership and fellowship behaviors and an increasing awareness [4].

Anesthesia was one of the first specialties to adopt the CRM training and an improvement of team performance and a reduced risk of errors during medical and surgical crisis in the operating room has been shown [5].

Early interest in this type of training was demonstrated also by the emergency medicine specialty.

Morey et al. (2002), in their evaluation of MedTeams Project's results found that formal teamwork training was effective in improving team behaviors and in reducing errors in Emergency Departments (ED) [6]. Despite this, human factors training is not widely disseminated through professionals working in ED and most studies focused only on emergency medicine residents and interdisciplinary trauma teams.

According to Chiniara (2003), simulation training is most suitable for low frequency high-risk situations that are potentially harmful for the patient [7]. These types of situations are those where crises arise, and CRM training may play an important role. As crises events are rare in real practice, the simulation room is an ideal setting for teaching CRM principles [7] and simulation-based CRM training was shown to be effective and relevant for the emergency medicine residents' practice [2].

Different scales were developed to assess the nontechnical skills/behavioral performance and many studies reported on the reaction of participants toward the training, the acquisition of knowledge and the changes in attitude in non-clinical settings. Only a limited number of studies focused on transfer of the learning to the working place and even less measured the effect on patient outcomes [8].

As morbidity and mortality are indicators difficult to track in prospective studies, surrogates such as time to disposition, delays in achieving tasks in a timeframe, number of errors [9] have been used as well as checklists, to assess the clinical performance.
This study is aimed at assessing whether a single day CRM oriented team training combining didactic and simulation sessions improves the clinical performance of interprofessional emergency medicine teams.

\section{口METHODS}

\section{Study design}

The study took place in the emergency department of the Tirgu-Mures Emergency Clinical County Hospital, Romania between March and July 2016. The hospital has an emergency medicine residency training program and is the site of an affiliated emergency and disaster medicine simulation center that focuses on multidisciplinary and inter-professional training. The emergency department's annual census is around 77,000 patients, approximately $10 \%$ of them being "critically ill".

Emergency department clinical staff without prior CRM training were invited to participate. Residents with less than three months clinical experience in the emergency department were excluded. Twenty boardcertified emergency medicine (EM) physicians, ten emergency medicine residents, and forty nurses volunteered for the study.

The study protocol was approved by the Ethics Committee of the Tirgu-Mures Emergency Clinical County Hospital. Written informed consent was obtained from all study participants. Twenty mixed teams were constructed according to participants' work schedules.

Each team utilized an attending EM physician, an EM resident and two nurses, a structure we consider ideal for the management of a single critically ill patient in our ED. Because the number of eligible attending physicians was greater than the number of residents, each resident participated in two teams.

Two sets of scenarios were designed, consisting of two scenarios each: a medical case and a trauma case.

The first set consisted of:

- Medical patient A - A patient with an acute COPD exacerbation complicated by severe pneumonia, initially requiring noninvasive and subsequently invasive ventilation. Due to patient physiognomy as well as a history of prior tracheostomy, a difficult intubation needed to be anticipated.

- Trauma patient A - A trauma patient with severe brain injury complicated by hemorrhagic shock secondary to intra-abdominal bleeding requiring 
activation of the massive transfusion protocol and emergent surgery.

The second set consisted of:

- Medical patient B - A patient with COPD now presenting with a pulmonary embolism necessitating thrombolysis due to clinical instability.

- Trauma patient B - A patient with a difficult airway, a severe, but surgically amenable intra-cranial injury, as well as long bones fractures, overall resulting in hypotension.

Both medical cases had signs of $\beta_{2}$ mimetics overuse (tremor, tachycardia, hypokalemia).

Sets were chosen pseudo-randomly, as participating residents were intended to be exposed to all cases and not to repeat scenarios. Simulations were performed in the ED resuscitation room during work hours. Scenarios utilized a high-fidelity manikin as well as the usual medical equipment and documentation forms from the resuscitation room. The role of different specialties doctors who were called for advice/help was played by one of the instructors, as well as the role of paramedics. The role of the radiologist was played by the radiologist on duty that day. Both a fixed camera, placed on the ceiling of the resuscitation room, as well as a mobile camera recorded all of the exercises. No debriefings took place after the assessment scenarios were completed.

We then held single day (6 to 7 hours) training sessions over a period of ten days. Participants chose the day for their training session at their convenience. Training sessions took place in a recreation of the ED resuscitation room in the simulation center. Each training session started with a lecture focusing on medical errors and CRM principles. No medical teaching was provided. After the lecture, participants had the opportunity to familiarize themselves with the manikin and the simulation setting.

Each training session involved two multi-professional teams, each team consisting of one EM attending physician, one EM resident and two nurses. During the training sessions participants were permitted to change teams as long as the multi-professional composition remained the same. We utilized six critical patient scenarios (two trauma cases and four medical cases), different from those used in the initial assessment. One team completed a scenario while the other team observed the exercise remotely via a high-resolution real time video transmission system. Either as participants or observers, each team was exposed to all cases.
The simulation was run by two instructors (one doctor and one nurse) with CRM background training and one IT technician. Each scenario was followed by an instructor facilitated debriefing. Both technical and non-technical issues relating to team performance and team work were discussed. Identified clinical errors were addressed by either the participants or the instructors. Members of the team who had completed the scenario had priority in providing feedback, but both observers and participants were involved in debriefings.

Two months after receiving training, a final assessment was performed consisting of the same scenarios, teams and setting used in the initial assessment. As in the initial assessment, the scenarios were video-recorded, and no briefing was provided.

Objective measurement of clinical team performance was performed through a checklist that was designed for each scenario (Figure 1). The checklist included essential assessment items for the diagnosis and treatment of a critical patient, with the focus on key actions and decisions. The number of times a critical procedure was performed, as well as time to completion of the critical steps where appropriate, were recorded.

The video recordings were analyzed, and the checklists completed by an assessor blinded to whether the scenarios analyzed were the initial or final assessment.

\section{Statistical analysis}

The collected data were organized into several SPSS data files. The variables obtained were binary type and continuous type. For binary data analysis, the likelihood ratio was used to assess the difference in proportions. Continuous variables were tested for normality using Kolmogorov-Smirnov test. Our data followed a non-gaussian distribution, therefore a non-parametric test was used to compare the central tendency for the data series. Wilcoxon signed rank test was used, since we analyzed paired data. The significance level used in all tests was 0.05 .

\section{RESULTS}

Seventy participants were enrolled in the study and 69 completed the study. One nurse participant resigned her position and she was not available for the final assessment scenario. She was replaced by another nurse who had attended the training. The initial nurse was excluded from the final analysis and the nurse who re- 


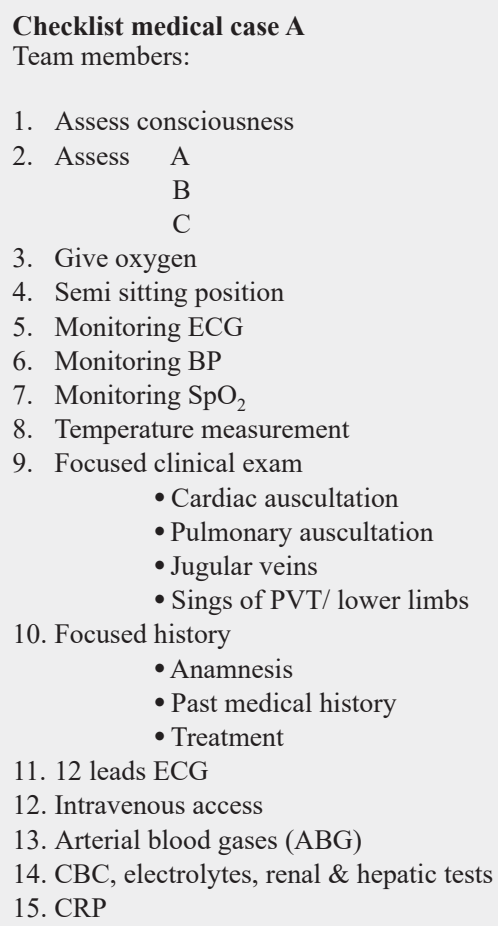

ig. 1. Example of a checklist used for the assessment of clinical performance

placed her was analyzed only once with the team to whom she was allocated at the beginning of the study.

The male: female ratio was 13:17 for doctors and 11:29 for nurses. Professional experience was variable (average 70 months, minimum 8 and maximum 300 months).

A checklist was completed for each scenario and each team. A total of 40 checklists were collected, 20 for the initial assessment and 20 the for the final one.

For the medical case A, the patient with acute COPD exacerbation complicated by severe pneumonia, critical elements where considered: oxygen administration, sitting position, establishing an intravenous access, appropriate investigations- especially ECG and arterial blood gases, recognition of the need for non-invasive ventilation, recognition of $\beta_{2}$ sympatico-mimetics overdose, appropriate antibiotic administration. Results are reported in table 1 and table 2 . A trend towards improvement was seen for these elements. Concerning the time until the procedure was performed, a statistically significant value was reached only for intravenous access. In terms of number of procedures performed, $\beta_{2}$ sympatico-mimetics overdose recognition and assessment of efficiency of NIV through repeated ABG 30 minutes after initiation of it, reached significance level.

\begin{tabular}{|c|c|c|}
\hline & & \\
\hline 16. NTproBNP & $\square$ & $\square$ \\
\hline 17. Troponin & $\square$ & $\square$ \\
\hline 18. D-dimers & $\square$ & $\square$ \\
\hline 19. Chest X-ray & $\square$ & $\square$ \\
\hline 20. Cardiac ultrasound & $\square$ & $\bar{\square}$ \\
\hline 21. Give $\beta_{2}$ mimetics & $\square$ & $\square$ \\
\hline 22. Noninvasive ventilation & $\square$ & $\square$ \\
\hline - Choose the mask & $\square$ & $\square$ \\
\hline - Set the ventilator correctly & $\square$ & $\square$ \\
\hline - Explain to the patient & $\square$ & $\square$ \\
\hline - Apply the mask & $\square$ & $\square$ \\
\hline - Observe for tolerance \&synchronism & $\square$ & $\square$ \\
\hline - Observe the patient & $\square$ & $\square$ \\
\hline - Observe the ventilator & $\square$ & $\square$ \\
\hline 23. Repeat ABG after 30 minutes & $\square$ & $\square$ \\
\hline 24. Give appropriate antibiotic & $\square$ & $\bar{\square}$ \\
\hline 25. Differential diagnosis & & \\
\hline - Exacerbation of COPD+ pneumonia & $\square$ & $\square$ \\
\hline - Acute pulmonary edema & $\square$ & $\square$ \\
\hline - Acute coronary syndrome & $\square$ & $\square$ \\
\hline - Pulmonary embolism & $\square$ & $\square$ \\
\hline - Spontaneous pneumothorax & $\square$ & $\square$ \\
\hline 26. Final diagnosis & & \\
\hline - Exacerbation of COPD+ pneumonia & $\square$ & $\square$ \\
\hline 27. Recognize $\beta_{2}$-mimetics overdose & $\square$ & $\square$ \\
\hline
\end{tabular}

For medical case B, the patient with COPD now presenting with a pulmonary embolism, critical elements where: oxygen administration, semi-sitting position, establishing an intravenous access, appropriate investigations- especially ECG, arterial blood gases, angioCT scan/ cardiac ultrasound, recognition of the need for and initiation of thrombolysis, request for cardiologic advice and recognition of $\beta_{2}$ sympatico-mimetics overdose. Results are reported in table 3 and table 4 . As for the medical case A, an improvement was seen for most of the elements. Significance level was reached in terms of number of procedures performed for cardiac ultrasound requested and $\beta_{2}$ sympatico-mimetics overdose recognition. Concerning the time until the procedure was performed, significance level was reached for oxygen administration and sitting position. For time until a cardiac ultrasound was requested, the $\mathrm{p}$ value couldn't be calculated as the test was requested during the initial assessment scenario only by two teams.

Critical elements for the trauma case A, the patient with severe brain injury complicated by hemorrhagic shock secondary to intra-abdominal bleeding, were considered: 2 intravenous access, FAST ultrasound examination, administration of fluid bolus and $\mathrm{O}$ negative PRBCs, recognition of a shock patient requiring urgent surgery (request for surgeon advise early, transfer to the 
Table 1. Checklist results for medical case A

\begin{tabular}{|c|c|c|c|c|c|c|}
\hline \multicolumn{2}{|l|}{ Element } & $\begin{array}{l}\text { Number of } \\
\text { teams } \\
\text { "Yes"/initial }\end{array}$ & $\begin{array}{c}\text { Number of } \\
\text { teams } \\
\text { "Yes"/final }\end{array}$ & $\begin{array}{c}\text { Number of } \\
\text { teams } \\
\text { "No"/initial }\end{array}$ & $\begin{array}{c}\text { Number of } \\
\text { teams } \\
\text { "No"/final }\end{array}$ & $\begin{array}{l}\text { Likelihood } \\
\text { Ratio* }\end{array}$ \\
\hline \multicolumn{2}{|l|}{ Consciousness } & 10 & 10 & 0 & 0 & \\
\hline \multicolumn{2}{|c|}{$\mathrm{ABC}$ evaluation $<2$ minutes } & 10 & 10 & 0 & 0 & \\
\hline \multicolumn{2}{|c|}{ Oxygen administration $* *$} & 10 & 10 & 0 & 0 & \\
\hline \multicolumn{2}{|c|}{ Sitting position $* *$} & 10 & 10 & 0 & 0 & \\
\hline \multirow[t]{4}{*}{ Monitoring } & Electrocardiogram & 10 & 10 & 0 & 0 & \\
\hline & Blood pressure & 10 & 10 & 0 & 0 & \\
\hline & Oxygen saturation & 10 & 10 & 0 & 0 & \\
\hline & Temperature & 8 & 9 & 2 & 1 & 0.528 \\
\hline \multirow{4}{*}{$\begin{array}{l}\text { Focused clinical } \\
\text { examination }\end{array}$} & Cardiac auscultation & 1 & 2 & 9 & 1 & 0.528 \\
\hline & Pulmonary auscultation & 10 & 10 & 0 & 0 & \\
\hline & Jugular veins & 0 & 1 & 10 & 9 & \\
\hline & Signs of PVT/edema & 5 & 10 & 5 & 0 & \\
\hline \multirow{3}{*}{ Focused history } & Anamnesis & 10 & 10 & 0 & 0 & \\
\hline & Past medical history & 10 & 10 & 0 & 0 & \\
\hline & Treatment & 8 & 10 & 2 & 0 & \\
\hline \multicolumn{2}{|c|}{ 12-lead electrocardiogram ** } & 6 & 10 & 4 & 0 & \\
\hline \multicolumn{2}{|c|}{ Intravenous line $* *$} & 10 & 10 & 0 & 0 & \\
\hline \multicolumn{2}{|c|}{ Arterial blood gases $(A B G) * *$} & 10 & 10 & 0 & 0 & \\
\hline \multirow[t]{5}{*}{ Lab tests } & CBC, electrolytes, Renal \&hepatic tests & 9 & 10 & 1 & 0 & \\
\hline & C-reactive protein & 1 & 0 & 9 & 10 & \\
\hline & NTproBNP & 8 & 2 & 8 & 2 & 1.000 \\
\hline & Troponin & 2 & 4 & 8 & 6 & 0.326 \\
\hline & D-dimers & 6 & 8 & 4 & 2 & 0.326 \\
\hline \multicolumn{2}{|c|}{ Chest radiography } & 10 & 10 & 0 & 0 & \\
\hline \multicolumn{2}{|c|}{ Cardiac ultrasound } & 1 & 1 & 9 & 9 & 1.000 \\
\hline \multicolumn{2}{|l|}{ Give $\beta_{2}$ mimetics } & 9 & 3 & 1 & 7 & 0.004 \\
\hline \multicolumn{2}{|c|}{ Noninvasive ventilation $* *$} & 10 & 10 & 0 & 0 & \\
\hline & Choose the mask & 8 & 7 & 2 & 3 & 0.605 \\
\hline & Set the ventilator correctly & 8 & 9 & 2 & 1 & 0.528 \\
\hline & Explain to the patient & 8 & 10 & 2 & 0 & 0.112 \\
\hline & Apply the mask & 10 & 10 & 0 & 0 & \\
\hline & Observe for tolerance \& synchronism & 6 & 9 & 4 & 1 & 0.112 \\
\hline & Observe the patient & 9 & 9 & 1 & 1 & 1.000 \\
\hline & Observe the ventilator & 7 & 9 & 3 & 1 & 0.255 \\
\hline \multicolumn{2}{|c|}{ Repeat ABG after 30 minutes } & 4 & 9 & 6 & 1 & 0.015 \\
\hline \multicolumn{2}{|c|}{ Give appropriate antibiotic } & 8 & 10 & 2 & 0 & \\
\hline \multirow{5}{*}{$\begin{array}{l}\text { Differential } \\
\text { diagnosis }\end{array}$} & Exacerbation of COPD+ pneumonia & 10 & 10 & 0 & 0 & \\
\hline & Acute pulmonary edema & 4 & 10 & 6 & 0 & \\
\hline & Acute coronary syndrome & 0 & 3 & 10 & 7 & \\
\hline & Pulmonary embolism & 7 & 10 & 3 & 0 & \\
\hline & Spontaneous pneumothorax & 2 & 6 & 8 & 4 & 0.063 \\
\hline \multicolumn{2}{|c|}{ Correct diagnosis } & 10 & 10 & 0 & 0 & \\
\hline \multicolumn{2}{|c|}{ Recognize $\beta_{2}$ mimetics overdose } & 1 & 7 & 9 & 3 & 0.004 \\
\hline
\end{tabular}

${ }^{*}$ Chi-Square Tests; ** Time to achievement recorded; ABC- airway, breathing, circulation, PVT-profound venous thrombosis, CBC- complete blood count, NTproBNP- N-terminal pro b-type natriuretic peptide, COPD-chronic obstructive pulmonary disease

Table 2. Results for medical case A - Time to achievement of critical steps

\begin{tabular}{|c|c|c|c|c|c|}
\hline \multirow{2}{*}{ Time to.... (seconds) } & \multicolumn{2}{|c|}{ Initial } & \multicolumn{2}{|c|}{ Final } & \multirow{2}{*}{ P value* } \\
\hline & Median & IQR & Median & IQR & \\
\hline Oxygen administration & 65 & 80 & 39.5 & 63 & 0.203 \\
\hline Sitting position & 17 & 113 & 6 & 18 & 0.074 \\
\hline 12 leads electrocardiogram & 56 & 121 & 136.5 & 111 & 0.463 \\
\hline Intravenous line & 123.5 & 114 & 58.5 & 54 & 0.013 \\
\hline Arterial blood gases & 142.5 & 99 & 70.5 & 99 & 0.169 \\
\hline Noninvasive ventilation & 308 & 304 & 215.5 & 124 & 0.386 \\
\hline
\end{tabular}


Table 3. Checklist results for medical case B

\begin{tabular}{|c|c|c|c|c|c|c|}
\hline \multicolumn{2}{|l|}{ Element } & $\begin{array}{c}\text { Number of } \\
\text { teams } \\
\text { "Yes"/initial }\end{array}$ & $\begin{array}{l}\text { Number of } \\
\text { teams } \\
\text { "Yes"/final }\end{array}$ & $\begin{array}{l}\text { Number } \\
\text { of teams } \\
\text { "No"/initial }\end{array}$ & $\begin{array}{l}\text { Number } \\
\text { of teams } \\
\text { "No"/final }\end{array}$ & $\begin{array}{l}\text { Likelihood } \\
\text { Ratio* }\end{array}$ \\
\hline \multicolumn{2}{|l|}{ Consciousness } & 10 & 10 & 0 & 0 & \\
\hline \multicolumn{2}{|c|}{$\mathrm{ABC}$ evaluation $<2$ minutes } & 10 & 10 & 0 & 0 & \\
\hline \multicolumn{2}{|c|}{ Oxygen administration $* *$} & 10 & 10 & 0 & 0 & \\
\hline \multicolumn{2}{|c|}{ Sitting position $* *$} & 9 & 10 & 1 & 0 & \\
\hline \multirow{4}{*}{ Monitoring } & Electrocardiogram & 10 & 10 & 0 & 0 & \\
\hline & Blood pressure & 10 & 10 & 0 & 0 & \\
\hline & Oxygen saturation & 10 & 10 & 0 & 0 & \\
\hline & Temperature & 6 & 1 & 4 & 9 & 0.015 \\
\hline \multirow{4}{*}{$\begin{array}{l}\text { Focused clinical } \\
\text { examination }\end{array}$} & Cardiac auscultation & 4 & 8 & 6 & 2 & 0.063 \\
\hline & Pulmonary auscultation & 9 & 8 & 1 & 2 & 0.528 \\
\hline & Jugular veins & 1 & 3 & 9 & 7 & 0.255 \\
\hline & Signs of PVT/edema & 9 & 10 & 1 & 0 & \\
\hline \multirow[t]{3}{*}{ Focused history } & Anamnesis & 10 & 10 & 0 & 0 & \\
\hline & Past medical history & 10 & 10 & 0 & 0 & \\
\hline & Treatment & 10 & 10 & 0 & 0 & \\
\hline \multicolumn{2}{|c|}{ 12-lead electrocardiogram** } & 10 & 10 & 0 & 0 & \\
\hline \multicolumn{2}{|c|}{ Intravenous line ** } & 10 & 10 & 0 & 0 & \\
\hline \multicolumn{2}{|c|}{ Arterial blood gases (ABG) } & 10 & 10 & 0 & 0 & \\
\hline \multirow[t]{5}{*}{ Lab tests } & CBC, electrolytes, Renal \&hepatic tests & 10 & 10 & 0 & 0 & \\
\hline & CRP & 0 & 0 & 10 & 10 & \\
\hline & NT-proBNP & 8 & 9 & 2 & 1 & 0.528 \\
\hline & Troponin & 9 & 8 & 1 & 2 & 0.528 \\
\hline & D-dimers & 9 & 10 & 1 & 0 & \\
\hline \multicolumn{2}{|c|}{ Chest radiography } & 6 & 4 & 4 & 6 & 0.369 \\
\hline \multicolumn{2}{|c|}{ Fluids bolus } & 2 & 9 & 8 & 1 & 0.001 \\
\hline \multicolumn{2}{|l|}{ Give $\beta$, mimetics } & 8 & 2 & 2 & 8 & 0.005 \\
\hline \multicolumn{2}{|c|}{ Request angioCT $\operatorname{scan} * *$} & 10 & 8 & 0 & 2 & \\
\hline \multicolumn{2}{|c|}{ Angio-CT scan performed } & 6 & 2 & 4 & 6 & 0.132 \\
\hline \multicolumn{2}{|c|}{ Request cardiac ultrasound $* *$} & 2 & 7 & 8 & 3 & 0.021 \\
\hline \multicolumn{2}{|c|}{ Request venous Doppler ultrasound } & 2 & 0 & 8 & 10 & \\
\hline \multicolumn{2}{|c|}{ Decide to thrombolysis $* *$} & 9 & 10 & 1 & 0 & \\
\hline \multicolumn{2}{|c|}{ Verify contraindications to thrombolysis } & 2 & 7 & 8 & 3 & 0.021 \\
\hline \multicolumn{2}{|c|}{ Start thrombolysis ** } & 9 & 10 & 1 & 0 & \\
\hline \multicolumn{2}{|c|}{ Ask for cardiologic advise $* *$} & 10 & 10 & 0 & 0 & \\
\hline \multirow{5}{*}{$\begin{array}{l}\text { Differential } \\
\text { diagnosis }\end{array}$} & Exacerbation of COPD+ pneumonia & 6 & 9 & 4 & 1 & 0.112 \\
\hline & Acute pulmonary edema & 4 & 5 & 6 & 5 & 0.653 \\
\hline & Acute coronary syndrome & 5 & 10 & 5 & 0 & \\
\hline & Pulmonary embolism & 10 & 10 & 0 & 0 & \\
\hline & Spontaneous pneumothorax & 5 & 8 & 5 & 2 & 0.155 \\
\hline \multicolumn{2}{|c|}{ Correct diagnosis } & 10 & 10 & 0 & 0 & \\
\hline Recognize $\beta_{2}$ mir & netics overdose & 2 & 8 & 8 & 2 & 0.005 \\
\hline
\end{tabular}

${ }^{*}$ Chi-Square Test; ** Time to achievement recorded; ABC- airway, breathing, circulation, PVT-profound venous thrombosis, CBC-complete blood count, NT-proBNP- N-terminal pro b-type natriuretic peptide, COPD-chronic obstructive pulmonary disease

Table 4. Results for medical case B - Time to achievement of critical steps

\begin{tabular}{|c|c|c|c|c|c|}
\hline \multirow[t]{2}{*}{ Time to.... (seconds) } & \multicolumn{2}{|c|}{ Initial } & \multicolumn{2}{|c|}{ Final } & \multirow{2}{*}{ P value* } \\
\hline & Median & IQR & Median & IQR & \\
\hline Oxygen administration & 76 & 81 & 51.5 & 29 & 0.022 \\
\hline Sitting position & 20 & 245 & 6.5 & 15 & 0.012 \\
\hline 12 leads electrocardiogram & 160 & 125 & 120 & 30 & 0.221 \\
\hline intravenous line & 115 & 116 & 105 & 48 & 0.859 \\
\hline Arterial blood gases & 159 & 73 & 128 & 144 & 0.767 \\
\hline AngioCT scan & 345 & 369 & 501 & NA & NA \\
\hline Cardiac ultrasound & 790 & NA & 520 & 450 & NA \\
\hline Thrombolysis decision & 515 & 233 & 510 & 394 & 0.515 \\
\hline Start thrombolysis & 570 & 673 & 606.5 & 410 & 0.678 \\
\hline Request cardiologic advice & 336 & 280 & 300 & 580 & 0.333 \\
\hline
\end{tabular}


operating theatre), recognition of the need to intubate and anticipation of a difficult airway, recognition and initiation of treatment for increased ICP. Results are reported in table 5 and table 6 . There was a trend towards improvement in all the elements, though not statistically significant, except for recognition and initiation of treatment of high ICP, and the time until airway was secured.

The trauma case $\mathrm{B}$, the patient with a difficult airway, severe TBI and long bones fractures resulting in hypotension, had the following elements: intravenous access, administration of fluid bolus, FAST ultrasound examination, recognition of severe brain injury requiring urgent measures to decrease the ICP, performance of the CT scan and early involvement of the neurosurgeon, recognition of the need to intubate, anticipation of a difficult airway and management of a "cannot ventilate, cannot intubate " situation (number of direct laryngoscopies, call for help, decide and perform a cri-

Table 5. Checklist results for trauma case A

\begin{tabular}{|c|c|c|c|c|c|c|}
\hline \multicolumn{2}{|l|}{ Element } & $\begin{array}{c}\text { Number } \\
\text { of teams } \\
\text { "Yes"/initial }\end{array}$ & $\begin{array}{l}\text { Number } \\
\text { of teams } \\
\text { "Yes"/final }\end{array}$ & $\begin{array}{l}\text { Number } \\
\text { of teams } \\
\text { "No"/initial }\end{array}$ & $\begin{array}{l}\text { Number } \\
\text { of teams } \\
\text { "No"/final }\end{array}$ & $\begin{array}{l}\text { Likelihood } \\
\text { Ratio* }\end{array}$ \\
\hline \multicolumn{2}{|l|}{ Consciousness } & 10 & 10 & 0 & 0 & \\
\hline \multirow{7}{*}{$\begin{array}{l}\text { Primary survey } \\
\text { ( } A B C<2 \text { minutes) } \\
\text { ( } D E<5 \text { minutes) }\end{array}$} & A-airway & 6 & 10 & 4 & 0 & \\
\hline & B-breathing & 9 & 10 & 1 & 0 & \\
\hline & C-circulation & 9 & 10 & 1 & 0 & \\
\hline & $\mathrm{D} \quad \mathrm{GCS}$ & 9 & 10 & 1 & 0 & \\
\hline & Motor response & 7 & 10 & 3 & 0 & \\
\hline & Pupillary exam & 9 & 10 & 1 & 0 & \\
\hline & E-exposure & 10 & 10 & 0 & 0 & \\
\hline \multicolumn{2}{|c|}{ Oxygen administration } & 10 & 10 & 0 & 0 & \\
\hline \multirow{4}{*}{ Monitoring } & Electrocardiogram & 10 & 10 & 0 & 0 & \\
\hline & Blood pressure & 10 & 10 & 0 & 0 & \\
\hline & Oxygen saturation & 10 & 10 & 0 & 0 & \\
\hline & Temperature & 1 & 5 & 9 & 5 & 0.044 \\
\hline \multirow{7}{*}{ Focused history } & Anamnesis & 10 & 10 & 0 & 0 & \\
\hline & Past medical history & 1 & 0 & 9 & 10 & \\
\hline & Allergies & 0 & 0 & 10 & 10 & \\
\hline & Treatment & 0 & 1 & 10 & 9 & \\
\hline & Mechanism of injury & 7 & 10 & 3 & 0 & \\
\hline & Time from injury & 0 & 7 & 10 & 3 & \\
\hline & Last meal & 0 & 1 & 10 & 9 & \\
\hline \multicolumn{2}{|c|}{ Insert an intravenous line $* *$} & 10 & 10 & 0 & 0 & \\
\hline \multicolumn{2}{|c|}{ ABO+ Rhesus } & 10 & 10 & 0 & 0 & \\
\hline \multicolumn{2}{|l|}{ Glucose level } & 2 & 1 & 8 & 9 & 0.528 \\
\hline \multicolumn{2}{|c|}{$\begin{array}{l}\text { CBC, electrolytes, renal \&hepatic, coagulation tests, } \\
\text { creatine kinase, arterial blood gases }\end{array}$} & 10 & 10 & 0 & 0 & \\
\hline \multicolumn{2}{|c|}{ Fluids bolus ** } & 7 & 10 & 3 & 0 & \\
\hline \multicolumn{2}{|c|}{ FAST ultrasound exam ** } & 10 & 10 & 0 & 0 & \\
\hline \multicolumn{2}{|c|}{ 12-lead electrocardiogram ** } & 3 & 4 & 7 & 6 & 0.639 \\
\hline \multicolumn{2}{|c|}{ Insert a second intravenous line $* *$} & 10 & 10 & 0 & 0 & \\
\hline \multicolumn{2}{|c|}{ Recognize signs of shock } & 10 & 10 & 0 & 0 & \\
\hline \multicolumn{2}{|c|}{ Transfusion of O negative PRBCs } & 6 & 9 & 4 & 1 & 0.121 \\
\hline \multicolumn{2}{|c|}{ Recognize signs of increased ICP } & 6 & 10 & 4 & 0 & \\
\hline \multicolumn{2}{|c|}{ Recognize the need for intubation ** } & 10 & 10 & 0 & 0 & \\
\hline \multicolumn{2}{|c|}{ Anticipate a difficult airway } & 3 & 7 & 7 & 3 & 0.070 \\
\hline \multicolumn{2}{|l|}{ Airway secured $* *$} & 10 & 10 & 0 & 0 & \\
\hline \multicolumn{2}{|c|}{ Request the surgeon advise $* *$} & 10 & 10 & 0 & 0 & \\
\hline \multicolumn{2}{|c|}{ Request for a CT scan } & 3 & 3 & 7 & 7 & 1.000 \\
\hline \multicolumn{2}{|c|}{ Request the neurosurgeon advise } & 5 & 8 & 5 & 2 & 0.155 \\
\hline \multicolumn{2}{|l|}{ Treat high ICP } & 2 & 7 & 8 & 3 & 0.021 \\
\hline Immobilize the frac & & 5 & 10 & 5 & 0 & \\
\hline Perform the logroll & & 1 & 3 & 9 & 7 & 0.255 \\
\hline Perform a complet & econdary survey & 1 & 0 & 9 & 10 & \\
\hline Transfer the patien & o the operating room $* *$ & 9 & 10 & 1 & 0 & \\
\hline IOT checklist comp & ed verified & 0 & 2 & 10 & 8 & \\
\hline
\end{tabular}


Table 6. Results for trauma case A - Time to achievement of critical steps

\begin{tabular}{|c|c|c|c|c|c|}
\hline \multirow{2}{*}{ Time to.... (seconds) } & \multicolumn{2}{|c|}{ Initial } & \multicolumn{2}{|c|}{ Final } & \multirow{2}{*}{ P value* } \\
\hline & Median & IQR & Median & IQR & \\
\hline First intravenous line & 65 & 49 & 43.5 & 45 & 0.139 \\
\hline Fluids bolus & 208 & 258 & 116.5 & 114 & 0.128 \\
\hline FAST ultrasound exam & 174 & 177 & 160.5 & 160 & 0.721 \\
\hline 12 leads electrocardiogram & 658 & NA & 114 & 52 & 0.109 \\
\hline Second intravenous line & 174 & 352 & 158.5 & 190 & 0.646 \\
\hline Recognize the need for IOT & 149 & 116 & 109 & 199 & 0.203 \\
\hline Airway secured & 401 & 210 & 317 & 78 & 0.017 \\
\hline Request the surgeon advise & 339.5 & 215 & 247.5 & 247 & 0.241 \\
\hline Transfer into the operating room & 580 & 295 & 605 & 70 & 0.374 \\
\hline
\end{tabular}

cothyroidotomy). Results are given in table 7 and table 8. An improvement was seen in most of the elements. Significant improvement was shown in the elements corelated with severe TBI and difficult airway management, in terms of number of procedures performed (recognition and treatment of increased ICP, anticipation of difficult airway) and time until procedure was completed (request for neurosurgeon advice, first attempt to intubate, decision for cricothyroidotomy, airway secured).

\section{DISCUSSION}

CRM trainings are addressing a set of nontechnical skills, "the cognitive, social and personal resource skills that complement technical skills, and contribute to safe and efficient task performance" [10]. These nontechnical skills can be divided into four domains essential for an effective team: teamwork, task management, decision making and situational awareness, all of them being linked by communication [11]

The CRM key principles that act as a framework for teaching teamwork skills to emergency residents, according to GABA [5] and modified by Carne [12] are:

- Know your environment

- Anticipate, share and review the plan

- Ensure leadership and role clarity

- Communicate effectively

- Call for help early

- Allocate attention wisely- avoid fixation

- Distribute the workload-monitor and support team members.

In our study we assessed whether a single-day training on CRM principles combining didactic and high-fidelity simulated session may improve the clinical performance (objectively measured through a checklist) of an interprofessional emergency team. We found that most of the measured clinical parameters improved. We hypothesize this is due to an improvement of nontechnical skills, as we didn't teach the clinical skills in the simulation cases explicitly. Also, in each team we had an experienced attending physician, the mean experience for the EM attendings being 149 months (minimum 72 and maximum 300 months), so lack of knowledge was unlikely.

Many of the clinical decisions as well as the evaluation of the patient include the correct usage of CRM principles.

The cases were chosen in such a way that they were typical for an average acute ED patient, but still strongly dependent on correct application of CRM principles. E.g., when dealing with a difficult airway, in our case a "cannot ventilate cannot intubate" situation, the team should anticipate, share the same mental model, distribute the workload and plan ahead while staying alert for sudden changes of situation. All the required skills correspond to CRM principles and indeed improved significantly after the training as shown by the number of teams who anticipated the difficult airway $(p=0.044)$, number of attempts to intubate by direct laryngoscopy, the time until decision to perform a cricothyroidotomy was taken and the airway was secured.

Managing a polytrauma patient with hemorrhagic shock, requiring damage control surgery and massive transfusion protocol activation, requires to: anticipate, share the plan, ensure leadership and role clarity, communicate effectively, distribute the workload, call for help early and maintain standards. An improvement was seen in the present study, reflected by number of teams that administrate fluids bolus and $\mathrm{O}$ negative PRBCs from the ED storage, monitored the temperature as hypothermia is one of the components of the lethal triad in bleeding patients [13], time until they insert the intravenous lines, gave the fluids bolus, performed the FAST exam, call for surgeon. 
Table 7. Checklist results for trauma case B

\begin{tabular}{|c|c|c|c|c|c|c|}
\hline \multicolumn{2}{|l|}{ Element } & $\begin{array}{c}\text { Number } \\
\text { of teams } \\
\text { "Yes"/initial }\end{array}$ & $\begin{array}{l}\text { Number } \\
\text { of teams } \\
\text { "Yes"/final }\end{array}$ & $\begin{array}{l}\text { Number } \\
\text { of teams } \\
\text { "No"/initial }\end{array}$ & $\begin{array}{l}\text { Number } \\
\text { of teams } \\
\text { "No"/final }\end{array}$ & $\begin{array}{c}\text { Likelihood } \\
\text { Ratio* }\end{array}$ \\
\hline \multicolumn{2}{|l|}{ Consciousness } & 10 & 10 & 0 & 0 & \\
\hline \multirow{7}{*}{$\begin{array}{l}\text { Primary survey } \\
\text { ( } A B C<2 \text { minutes) } \\
\text { ( } D E<5 \text { minutes) }\end{array}$} & A-airway & 9 & 10 & 1 & 0 & \\
\hline & B-breathing & 9 & 10 & 1 & 0 & \\
\hline & C-circulation & 9 & 10 & 1 & 0 & \\
\hline & $\mathrm{D} \quad \mathrm{GCS}$ & 8 & 10 & 2 & 0 & \\
\hline & Motor response & 7 & 10 & 3 & 0 & \\
\hline & Pupillary exam & 8 & 10 & 2 & 0 & \\
\hline & E-exposure & 10 & 10 & 0 & 0 & \\
\hline \multicolumn{2}{|c|}{ Oxygen administration } & 8 & 10 & 2 & 0 & \\
\hline \multirow{4}{*}{ Monitoring } & Electrocardiogram & 10 & 10 & 0 & 0 & \\
\hline & Blood pressure & 10 & 10 & 0 & 0 & \\
\hline & Oxygen saturation & 10 & 10 & 0 & 0 & \\
\hline & Temperature & 0 & 5 & 10 & 5 & \\
\hline \multirow{7}{*}{ Focused history } & Anamnesis & 10 & 10 & 0 & 0 & \\
\hline & Past medical history & 1 & 5 & 9 & 5 & 0.044 \\
\hline & Allergies & 0 & 7 & 10 & 3 & \\
\hline & Treatment & 0 & 1 & 10 & 9 & \\
\hline & Mechanism of injury & 7 & 10 & 3 & 0 & \\
\hline & Time from injury & 5 & 10 & 5 & 0 & \\
\hline & Last meal & 0 & 0 & 10 & 10 & \\
\hline \multicolumn{2}{|c|}{ Insert an intravenous line $* *$} & 10 & 10 & 0 & 0 & \\
\hline $\mathrm{ABO}+$ Rhesus & & 10 & 10 & 0 & 0 & \\
\hline Glucose level & & 2 & 3 & 8 & 7 & 0.605 \\
\hline $\begin{array}{l}\text { CBC, electrolytes, } r \\
\text { creatine kinase, art }\end{array}$ & $\begin{array}{l}\text { renal \&hepatic\& coagulation tests, } \\
\text { terial blood gases }\end{array}$ & 10 & 10 & 0 & 0 & \\
\hline Fluids bolus $* *$ & & 3 & 3 & 7 & 7 & 1.000 \\
\hline FAST ultrasound ex & xam ** & 5 & 7 & 5 & 3 & 0.359 \\
\hline 12-lead electrocarc & diogram & 1 & 4 & 9 & 6 & 0.112 \\
\hline Insert a second intr & ravenous line $* *$ & 8 & 8 & 2 & 2 & 1.000 \\
\hline Request for CT scar & & 9 & 10 & 1 & 0 & \\
\hline Request the neuros & surgeon ** & 9 & 10 & 1 & 0 & \\
\hline Recognize signs of & increased ICP & 3 & 9 & 7 & 1 & 0.004 \\
\hline Recognize the need & d for intubation $* *$ & 10 & 10 & 0 & 0 & \\
\hline Anticipate a difficu & It airway & 5 & 9 & 5 & 1 & 0.044 \\
\hline Number of direct la & aryngoscopy attempts (more than two) & 6 & 0 & 4 & 10 & \\
\hline Ventilation achieve & ed between intubation attempts & 10 & 10 & 0 & 0 & \\
\hline Ask for help ** & & 5 & 5 & 5 & 5 & 1.000 \\
\hline Use of alternative $t$ & techniques for intubation & 5 & 0 & 5 & 10 & \\
\hline Decide for cricothy & roidotomy ** & 10 & 10 & 0 & 0 & \\
\hline Airway secured ** & & 10 & 10 & 0 & 0 & \\
\hline Treat high ICP & & 4 & 9 & 6 & 1 & 0.015 \\
\hline Immobilize the frac & cture & 8 & 9 & 2 & 1 & 0.528 \\
\hline Give antibiotic & & 4 & 10 & 6 & 0 & \\
\hline Perform the logroll & & 4 & 8 & 6 & 2 & 0.063 \\
\hline Perform a complet & e secondary survey & 3 & 1 & 7 & 9 & 0.255 \\
\hline IOT checklist comp & leted verified & 0 & 2 & 10 & 8 & \\
\hline
\end{tabular}

Also, the recognition of a condition such as $\beta_{2}$ sympatico-mimetics overdose, in a patient presenting with respiratory distress, where signs as tachycardia and agitation might have different causes, request situational awareness and wise allocation of attention. In both medical cases, a significant improvement was seen after the training $(\mathrm{p}=0.004$ for case $\mathrm{A}$ and $\mathrm{p}=0.005$ for case B).

Avoiding fixation error was important in order to avoid a wrong diagnosis in medical cases as

they had a similar history and almost the same clinical presentation, except for the blood pressure that was 
Table 8. Results for trauma case B - Time to achievement of critical steps

\begin{tabular}{|c|c|c|c|c|c|}
\hline \multirow{2}{*}{ Time to.... (seconds) } & \multicolumn{2}{|c|}{ Initial } & \multicolumn{2}{|c|}{ Final } & \multirow{2}{*}{ P value* } \\
\hline & Median & IQR & Median & IQR & \\
\hline First intravenous line & 66 & 85 & 69.5 & 80 & 0.674 \\
\hline Fluids bolus & 245 & 703 & 114 & 188 & 0.317 \\
\hline FAST ultrasound exam & 170 & 875 & 240 & 510 & 0.715 \\
\hline Second intravenous line & 150 & 207 & 195 & 552 & 0.917 \\
\hline Request for neurosurgeon & 1200 & 338 & 580 & 465 & 0.008 \\
\hline Recognize the need for IOT & 240 & 175 & 145 & 80 & 0.017 \\
\hline First IOT attempt & 512 & 179 & 355 & 147 & 0.007 \\
\hline Call for help & 682 & 356 & 520 & 186 & 0.655 \\
\hline Decide for cricothyroidotomy & 790 & 195 & 446,5 & 262 & 0.005 \\
\hline Airway secured & 1030 & 304 & 602 & 250 & 0.005 \\
\hline
\end{tabular}

lower but still in normal range initially, for the patient with PE. Their final diagnosis was correct before and after the training, but more differential diagnoses were considered during the final assessment.

The idea of team skills training being associated with better clinical performance and empirically with better patient outcome was already raised by Wright et al [14]. In their study on medical students, run in two settings: a classroom-based and a high-fidelity simulated environment, they found a strong correlation between team skills rating and objective performance measures within the high fidelity simulated environment, but no correlation on the classroom- based environment.

Very few studies measured the impact of CRM training on patient outcome [8]. One of these found a significant decrease in mortality for in hospital pediatric cardiac arrest after simulation CRM training. [15].

A positive association between better teamwork, better nontechnical skills and disposition time and risk for delays to patient care was demonstrated by Pucher et al, on a study carried on fifty real trauma cases [9].

Similar to our study, the efficiency of a relatively brief (4 hours) teamwork training, including a onehour web didactic session followed by a human patient simulator-based session, on clinical performance of an interdisciplinary trauma team was shown by Steinmann et al [16]. This improvement was seen on simulated cases as well as on real trauma cases.

The effectiveness of teamwork training on clinical performance of an interprofessional team (surgeons, nurses), reflected by the decrease in time from arrival to CT scanner, endotracheal intubation and operating room, was demonstrated also by Capella et al [17]. As in the previously mentioned study, these results were obtained from real cases.

The present study has several limitations. The study was run in a single center and this limited the number of participants. Due to the very small number of EM residents available during the study period, they were exposed twice to the initial and final assessment in two different teams. Although we used two sets of scenarios and we did not perform a debriefing afterwards, we cannot exclude that their experience with the first team influenced their performance with the second one. It is difficult to appreciate if this had an impact the team clinical performance.

Participants were evaluated two months after the training. We did not assess them immediately after the training, so we do not know if their performance improved or declined over this time period.

Ideally, after a CRM training the actual patient outcome would be measured. This was our initial intention when we implemented this study. Unfortunately, we encountered a technical problem when we tried to analyze real cases. The quality of the image returned by the fixed camera was good, but the quality of the sound was too poor to permit a proper evaluation.

\section{CONCLUSION}

Our study supports the use of combined CRM training for improving clinical performance of an interprofessional emergency team. Empirically this may improve the patient outcome, but further research is necessary to establish the ideal length of the training, the real impact on patient outcome, the length of the decay phase and the optimal method and time for refresher training. 


\section{ACKNOWLEDGEMENTS}

The authors thank all the participants in the study. Special thanks to Oana Mates, Andreea Soceanu and Bogdan Posteuca for their help in collecting the data.

\section{CONFLICT OF INTEREST}

None to declare

\section{REFERENCES}

1. Rovamo L, Nurmi E, Mattila MM, et al. Effect of a simulationbased workshop on the multidisciplinary teamwork of newborn emergencies: an interventional study. BMC Res Notes. 2015;8:671.

2. Hicks MC, Kiss A, Bandiera WG, et al. Crisis Resources for Emergency Workers (CREW II): results of a pilot study and simulation-based crisis resource management course for emergency medicine residents. CJEM. 2012;14(6):354-62.

3. Petrosoniak A, Hicks CM. Beyond crisis resource management: new frontiers in human factors training for acute care medicine. Curr Opin Anesthesiol. 2013;26:699-706.

4. Rall M, Gaba D- Human Performance and Patient Safety. In Miller, RD (ed): Miller's Anesthesia. Philadelphia: Elsevier Churchill Livingstone. 2005, pp. 3021-72.

5. Gaba D, Howard S, Fish K. Simulation-based training in anesthesia crisis resource management (ACRM): a decade of experience. Simul Gaming. 2001;32:175-93.

6. Morey JC, Simon R, Jay GD, et al. Error Reduction and Performance Improvement in the Emergency Department through Formal Teamwork Training: Evaluation Results of the MedTeams Project. Health Serv Res. 2002;37(6):1553-81.
7. Chiniara G, Cole G, Brisbin K, et al. Simulation in healthcare: a taxonomy and a conceptual framework for instructional design and media selection. Med Teach. 2013;35(8):e1380-95.

8. Boet $S$, Bould MD, Fung $L$, et al. Transfer of learning and patient outcome in simulated crisis resource management: a systematic review. Can J Anesth. 2014;61:571-82.

9. Pucher PH, Aggarwal R, Batrick N, et al. Nontechnical skills performance and care processes in the management of the acute trauma patient. Surgery. 2014;155(5):902-9.

10. Flin R, O'Connor R, Crichton M. Safety at the Sharp End: A Guide to Nontechnical Skills. Farnham, UK: Ashgate; 2008.

11. Bleetman A, Sanusi S, Dale T, Brace S. Human factors and error prevention in emergency medicine. Emerg Med J. 2012;29:389-93.

12. Carne B, Kennedy M, Gray T. Crisis resource management in emergency medicine. Emerg Med Australas. 2012;24:7-13.

13. Simmons JW, Powell MF. Acute traumatic coagulopathy: pathophysiology and resuscitation. $\mathrm{Br} J$ Anaesth. 2016;117(3):iii31-iii43.

14. Wright MC, Phillips-Bute BG, Petrusa ER, Griffin KL, Hobbs GW, Taekman JM. Assessing teamwork in medical education and practice: relating behavioural teamwork ratings and clinical performance. Med Teach. 2009;31:30-8.

15. Andreatta P, Saxton E, Thompson M, Annich G. Simulationbased mock codes significantly correlate with improved pediatric cardiopulmonary arrest survival rates. Pediatr Crit Care Med. 2011;12:33-8.

16. Steinemann S, Berg B, Skinner A et al. In situ-multidisciplinary, simulated-based teamwork training improves early trauma care. J Surg Educ. 2011;68:472-7.

17. Capella J, Smith S, Philp A et al. Teamwork training improves the clinical care of trauma patients. J Surg Educ. 2010;67:43943. 